\title{
OPTICAL ACTUATION OF MICROFLUIDICS BASED ON OPTO-ELECTROWETTING
}

\author{
Pei Yu Chiou and Ming C. Wu \\ Department of Electrical Engineering, University of California at Los Angeles \\ Los Angeles, CA 90095-1594, USA
}

\author{
Hyejin Moon and Chang-Jin Kim \\ Department of Mechanical and Aerospace Engineering, \\ University of California at Los Angeles \\ Los Angeles, CA 90095-1594,USA
}

\author{
Hiroshi Toshiyoshi \\ Institute of Industrial Science, University of \\ Tokyo, Japan
}

\begin{abstract}
$^{1}$
Optical actuation of liquid droplets has been experimentally demonstrated for the first time using a novel opto-electrowetting principle. The opto-electrowetting (OEW) surface is realized by integrating a photoconductive material underneath a twodimensional array of electrowetting electrodes. Contact angle change as large as $40^{\circ}$ has been achieved when illuminated by a light beam with an intensity of $65 \mathrm{~mW} / \mathrm{cm}^{2}$. A micro-liter droplet of deionized water has been successfully dragged by a 4-mW laser beam across a $1 \mathrm{~cm} \times 1 \mathrm{~cm}$ OEW surface. The droplet speed is measured to be $7 \mathrm{~mm} / \mathrm{sec}$. Light actuation enables complex microfluidic functions to be performed on a single chip without encountering the wiring bottleneck of two-dimensional array of electrowetting electrodes.
\end{abstract}

\section{INTRODUCTION}

Surface tension is a dominant force for liquid handling and actuation in microscale. Several mechanisms have been proposed to control surface tension, including thermocapillary [1], electrowetting [2], and light-induced surface tension change [3]. Among them, the electrowetting mechanism has received increasing interests because of its fast switching response and low power consumption. The surface tension between the liquid-solid interface is modified by external electric field, which reduces the contact angle. Examples of electrowetting-based microfluidic systems include optical switches [4], digital microfluidic circuits [5] and liquid lenses with variable focal length [6].

Transport of liquid in droplet forms offers many advantages. It eliminates the need for pumps and valves, has no moving parts, and is free of leak and unwanted mixing. For Lab-on-a-Chip applications, several fluidic functions, such as liquid injection, transportation, mixing, and separation, need to be integrated on a single chip. This has been achieved by Cho, et al., recently [5]. For a general purpose fluidic chip that is capable of manipulating multiple droplets simultaneously requires a two-dimensional array of electrodes to control the local surface tension. However, this results in a large number of electrodes that presents a challenge for control and packaging of such chips. The problem becomes even more severe as the droplet size scales down. Though the number of electrodes can in principle be reduced by integrating address decoders on the chip, similar to the memory access circuits, this will significantly increase the cost of the chip.

Travel support has been generously provided by the Transducers Research Foundation and by the DARPA MEMS and DARPA BioFlips programs.
In this paper, we report on a novel mechanism for lightactuation of liquid droplets. This is realized by integrating a photoconductive material underneath the electrowetting electrodes. We called this mechanism "opto-electrowetting". We have successfully fabricated a prototype chip with $1 \mathrm{~cm} \times 1 \mathrm{~cm}$ area. A micro-liter droplet has been successfully transported to any location on the chip. This approach completely eliminates the wiring bottleneck of conventional electrowetting schemes. This concept is extendable to nano-liter droplets (or smaller) and simultaneously manipulation of multiple droplets.

\section{PRINCIPLE OF OPTO-ELECTROWETTING}

Figure 1(a) shows the general electrowetting mechanism. A droplet of polarizable and conductive liquid is placed on a substrate with an insulating layer between the liquid and the electrode. When an external voltage is applied, the surface tension at the solid-liquid interface is modified and the contact angle changes. The voltage dependence of the contact angle is described by Eq. (1):

$$
\cos \left[\theta\left(\boldsymbol{V}_{\boldsymbol{A}}\right)\right]=\cos [\theta(0)]+\frac{1}{2} \frac{\varepsilon}{\boldsymbol{d} \gamma_{L V}} \boldsymbol{V}_{\boldsymbol{A}}^{2}
$$

(a)

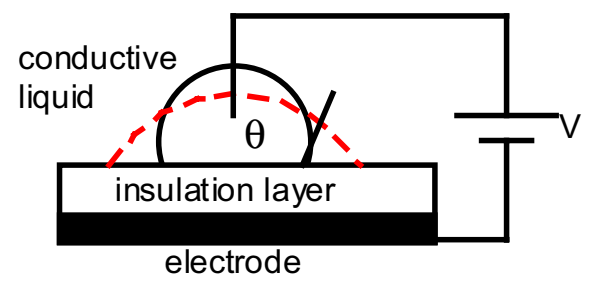

(b)

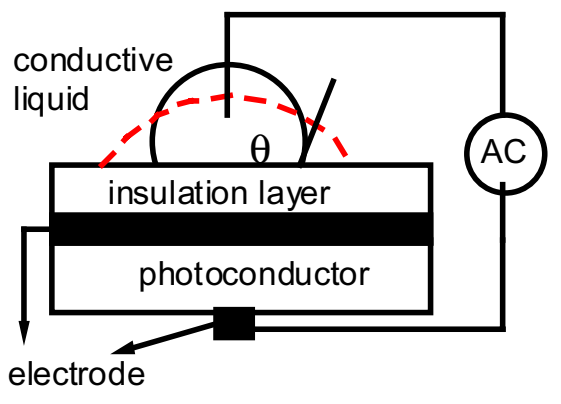

Figure.1 (a) Conventional electrowetting under DC bias, and (b) opto-electrowetting with an integrated photoconductor under $A C$ bias. 
where $\mathrm{V}_{\mathrm{A}}, \mathrm{d}, \varepsilon$, and $\gamma_{\mathrm{LV}}$ are applied voltage, thickness of insulating layer, dielectric constant of the insulating layer, and the interfacial tension between liquid and vapor, respectively. If an AC voltage is applied, $\mathrm{V}_{\mathrm{A}}$ is replaced by the root-mean-square (RMS) voltage [7].

Figure 1(b) shows the concept of the proposed optoelectrowetting (OEW) mechanism. A photoconductive material is integrated under the electrode of conventional electrowetting circuit. The electrical impedance of the liquid, insulator, and photoconductor are serially connected. From Eq. (1), the contact angle change of the droplet on this OEW surface is determined by the voltage drop across the insulating layer.

The frequency of the AC voltage is adjusted such that the impedance of the photoconductor dominates in the absence of light (dark state). Through the voltage divider, most of the voltage drops across the photoconductor and there is very little voltage across the insulating layer. Therefore, the contact angle remains the same as the equilibrium value. Upon illumination of light, the conductivity of the photoconductor increases by several orders of magnitude due to electron-hole pair generation. The impedance of the photoconductive layer becomes much smaller than that of the insulation layer. As a result, most of the voltage drop is now across the insulating layer. The contact angle is therefore reduced by light illumination.

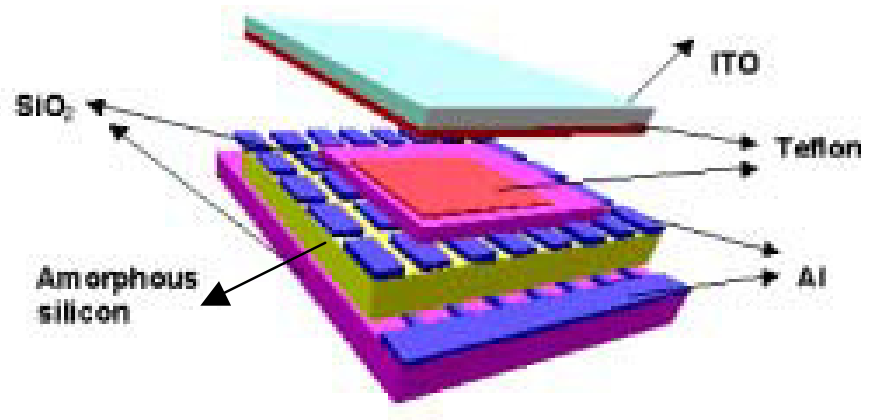

Fig.2 Schematic structure of the opto-electrowetting (OEW) device. A photoconductive material (amorphous Si) is integrated with a matrix electrode array to realize OEW mechanism.

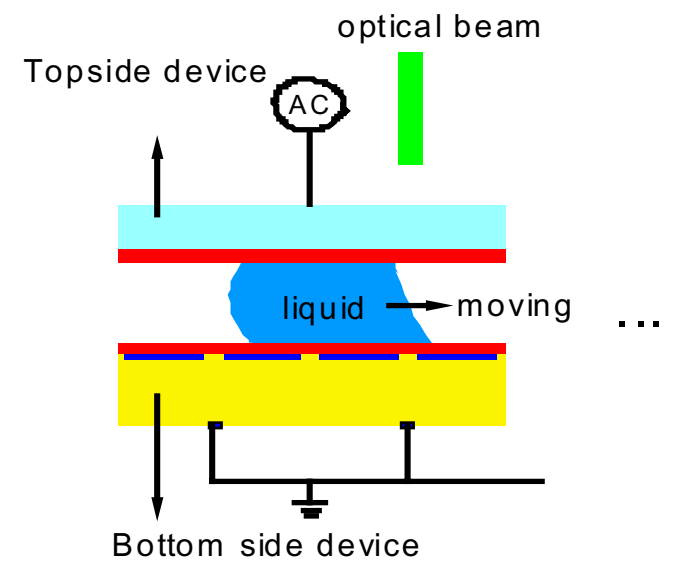

Fig.3 Principle illustrating light actuation of liquid droplets.
Figure 2 shows the schematic of the OEW device proposed in this paper. The liquid droplet is sandwiched between a top hydrophobic surface and a bottom OEW surface. The topside is a transparent conductive indium-tin-oxide (ITO) glass coated with $20 \mathrm{~nm}$ of Teflon. The OEW structure is realized by integrating a 2$\mathrm{D}$ array of electrowetting electrodes on a photoconductive material. In our current design, twenty thousand electrodes with areas of $50 \mu \mathrm{m} \times 100 \mu \mathrm{m}$ are employed over a total chip area of 1 $\mathrm{cm}^{2}$. The photoconductive material employed here is amorphous silicon. It is deposited by plasma-enhanced chemical vapor deposition (PECVD). The electrodes are then covered by a 0.9 $\mu$ m-thick $\mathrm{SiO}_{2}$ and a 20 -nm-thick Teflon. The $\mathrm{Al}$ electrode below the photoconductor has a grid pattern to reduce parasitic capacitance.

The moving principle and the equivalent circuit of this device is presented in Fig. 3 and 4, respectively. An AC voltage is applied between the top ITO electrode and the bottom $\mathrm{Al}$ grid. The voltage drop across the insulator and the electrode is controlled by light. Shining an optical beam on one edge of the liquid droplet decreases the contact angle and creates a pressure difference between two ends of the droplet, as shown in Fig. 3. This forces the droplet to follow the movement of the optical beam. Liquid droplet does not move for DC voltage bias because all the voltage drops across the insulating layer on every electrode even without light illumination. The contact angle on both ends of the droplet decreases and no pressure difference is created.

According to the equivalent circuit in Fig. 4, every electrode is connected in parallel. Voltage drop across the $\mathrm{SiO}_{2}$ on top of each electrode is a function of AC frequency and photoconductivity.

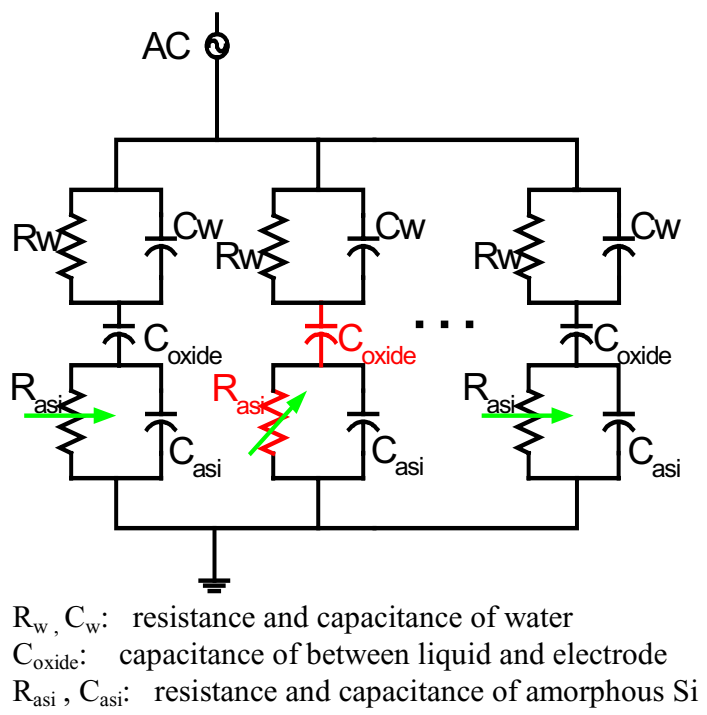

Fig.4 Equivalent circuit of the opto-electrowetting device. Under light illumination, the voltage drop across the photoconductor decreases while the voltage drop across $\mathrm{SiO}_{2}$ increases.

\section{CONTACT ANGLE MEASUREMENT UNDER LIGHT ILLUMINATION}

To effectively change the contact angle by light, the photoconductor needs to satisfy the following criteria:

(1) Low dark conductivity. This ensures the voltage will not drop across the insulator in the dark. 
(2) Short electron-hole pair recombination time. This will shorten the switching time

(3) Visible light response. Low cost visible light sources (either diode lasers or light-emitting diodes) are readily available.

Amorphous silicon satisfies all three criteria. It has a dark conductivity of $10^{-8} \mathrm{~S} / \mathrm{cm}$, an electron-hole pair recombination time of $\sim$ a microsecond, and is responsive to visible light. Figure 5(a) shows the experimental setup for measuring the photoconductivity. Two coplanar Al electrodes form Ohmic contacts with amorphous silicon. White light source with uniform intensity is used for measuring the photoconductivity.

The relationship between the photoconductivity and light intensity is shown in Fig. 6 . The conductivity increases by 80 times under illumination of light with an intensity of $65 \mathrm{mw} / \mathrm{cm}^{2}$.

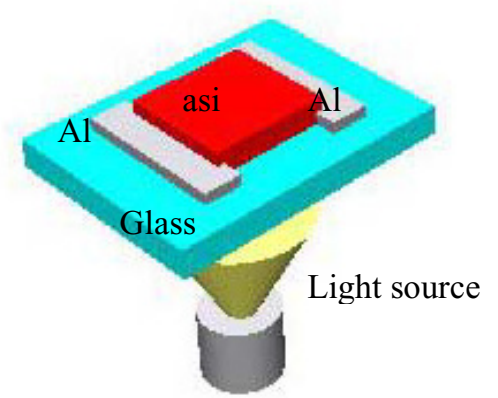

(a)

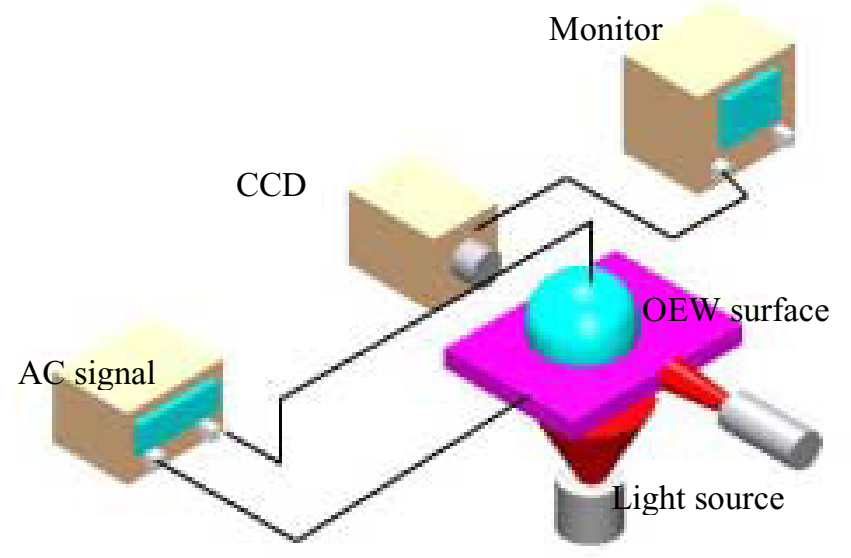

(b)

Fig.5 Experimental setup for (a) photoconductivity measurement, and (b) contact angle measurement on opto-electrowetting (OEW) surface.

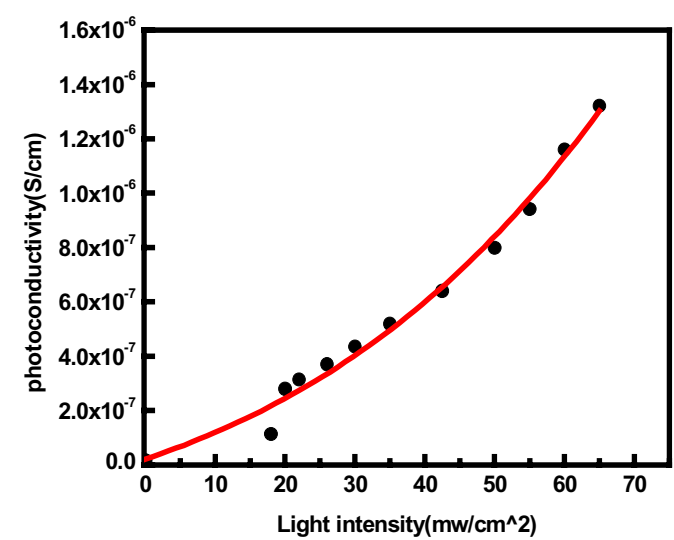

Fig.6 Photoconductivity versus light intensity for amorphous silicon..

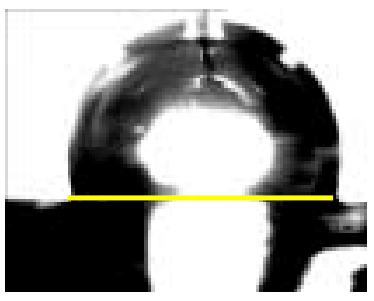

Without light

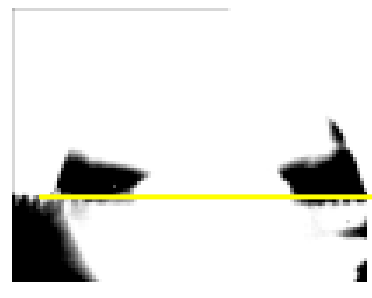

Light intensity: $65 \mathrm{mw} / \mathrm{cm}^{2}$
Fig. 7 Side-view profiles of the droplet with and without light illumination.

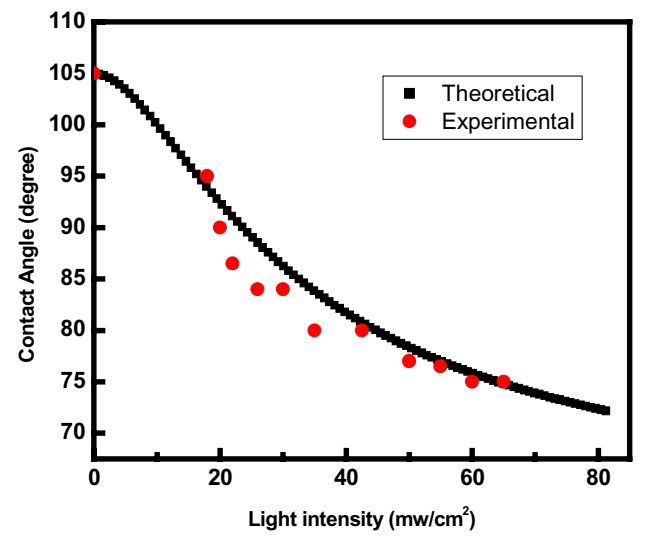

Fig.8 Contact angle change under light illumination

To measure the contact angle change, an OEW surface is manufactured on a glass substrate. The thickness of the amorphous silicon layer is the same as the sample used in the photoconductivity measurement. The experimental setup is shown in Fig. 5(b). The liquid used here is DI water. A CCD camera is used to image the side-view profile of the droplet. A $500 \mathrm{~Hz}, 100$ Volt AC signal is applied. Figure 7 shows the side-view profiles of the droplet in the dark and under light illumination of $65 \mathrm{mw} / \mathrm{cm}^{2}$ intensity. The theoretical calculation and experiment result are shown in Fig. 8. In our theoretical calculation, the conductivity of water is assumed to be $10^{-6} \mathrm{~S} / \mathrm{cm}$ and photoconductivity is 
assumed to have linear relationship with light intensity. In this device, the contact angle saturates at around 75 degrees. This saturation comes from the limitation of electrowetting.

\section{EXPERIMENTAL DEMONSTRATION OF LIQUID TRANSPORT BY LIGHT}

Figure 9 shows the photograph and microscope picture of the OEW device. The chip area is $1 \mathrm{~cm} \times 1 \mathrm{~cm}$, and the $\mathrm{Al}$ electrode area is $50 \mu \mathrm{m} \times 100 \mu \mathrm{m}$. Over 20,000 electrodes have been fabricated on the OEW surface. To demonstrate light actuation, a droplet with a diameter of $2 \mathrm{~mm}$ is sandwiched between a Tefloncoated ITO glass and an OEW surface with a gap of $0.5 \mathrm{~mm}$. A 4mw laser at $532 \mathrm{~nm}$ wavelength is used to drag the liquid droplet. The droplet is successfully moved across the $1 \mathrm{~cm} \times 1 \mathrm{~cm}$ surface by the laser beam. A droplet speed of $7 \mathrm{~mm} / \mathrm{s}$ was observed. Figure 10 shows four snap shots from the video recording of liquid transport.

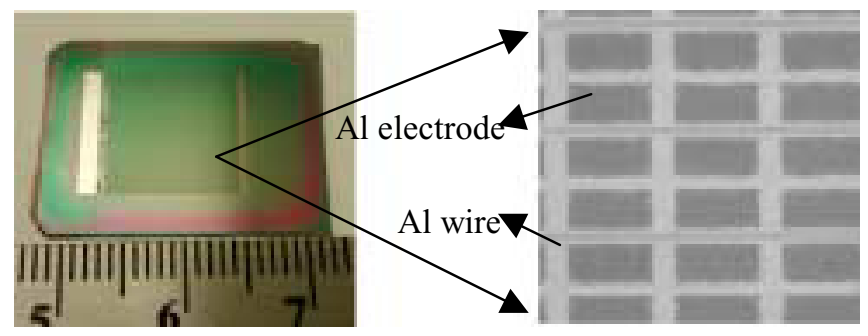

Fig.9 Photograph and microscope picture of the OEW device.
1

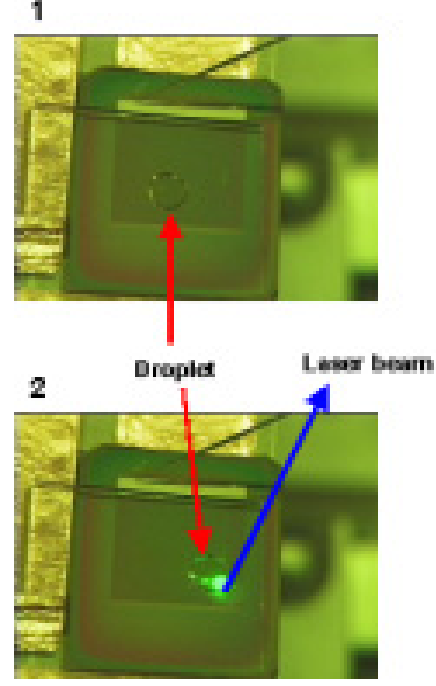

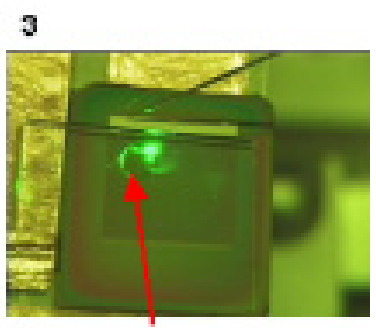

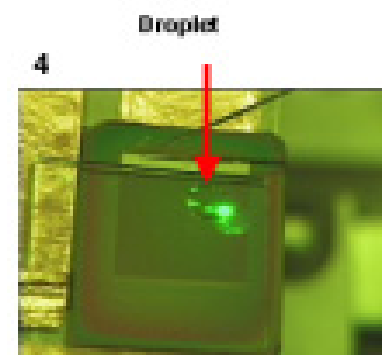

Fig.10 Example of liquid transport across a $1 \mathrm{~cm} \times 1 \mathrm{~cm}$ OEW area actuated by an optical beam.

\section{CONCLUSION}

A novel opto-electrowetting (OEW) mechanism has been proposed to move liquid droplets by light. This mechanism, combining electrowetting with light response of photoconductors. The light actuation enables a large number of electrowetting electrodes to be addressed without wiring bottlenecks.
Experimentally, over twenty thousand electrodes have been integrated on a $1 \mathrm{~cm} \times 1 \mathrm{~cm}$ area and only a single bias is needed for the entire device. Amorphous silicon is chosen to be the photoconductive material because of its properties of low dark conductivity, photoconductivity at visible wavelength, and short electron-hole pair recombination time. Under an AC bias voltage, the contact angle of the liquid droplet on this OEW surface is a function of light intensity. A 40 degrees contact angle change has been experimentally observed between dark and $65 \mathrm{mw} / \mathrm{cm}^{2}$ light illumination. The contact angle decreasing saturates at 75 degree in the OEW surface due to the wetting limitation of electrowetting. Movement of liquid droplet at $7 \mathrm{~mm} / \mathrm{sec}$ across the entire surface has been successfully demonstrated using a $4 \mathrm{~mW}$ laser beam.

\section{ACKNOWLEDGMENTS}

The authors would like to thank Sagi Mathai and Jui-Che Tsai for assisting our measurement, Pamela Peterson for valuable discussions of fabrication process, and professor Chih-Ming Ho for valuable discussions. This project is supported in part by DARPA Optoelectronics Center through CHIPS (Center for Heterogeneously Integrated Photonics) under contract \# MDA97200-1-0019.

\section{REFERENCES}

1. T.A. Sammarco, M.A. Burns, "Thermocapillary pumping of discrete drops in microfabricated device," AIChE J. 45 (2) (1999) 350-366.

2. Pollack, M.G.; Fair, R.B.; Shenderov, A.D., "Electrowettingbased actuation of liquid droplets for microfluidic applications," Applied Physics Letters, vol.77, (no.11), AIP, 11 Sept. 2000. p.1725-6.

3. Kunihiro Ichimura, Sang-Keun Oh, and Masaru Nakagawa, "Light-Driven Motion of Liquids on a Photoresponsive Surface," Science 2000 June 2; 288: 1624-1626

4. G.Beni, S. Hackwood, J. L. Jackel, "Continuous electrowetting effect”, Appl. Phys. Lett. 40 (10) (1982) 912-914.

5. S. K. Cho, S. K. Fan, Hyejin Moon, Chang-Jin Kim, "Towards digital microfluidic circuits: creating, transporting, cutting, and merging liquid droplets by electrowetting-based actuation," Technical Digest. MEMS 2002. 15th IEEE International Conference on Micro Electro Mechanical Systems, page 32-35.

6. Sunghoon Kwon, Luke P. Lee, "Focal lengthcontrol by microfabricated planar electrodes-based liquid lens(uPELL)," Digest of Technical Papers ,Transducers '01 Eurosensors XV $11^{\text {th }}$ International Conference on Solid-State Sensors and Actuactors,, page 1348-1351.

7. Vallet, M.; Vallade, M.; Berge, B. "Limiting phenomena for the spreading of water on polymer films by electrowetting," European Physical Journal B, vol.11, (no.4), EDP Sciences; Springer-Verlag, Oct. 1999. p.583-91. 